\title{
Modern Technologies for Level Measurement
}

Brumbi, Detlef, Prof. Dr.-Ing.

University of Applied Sciences

Edlmairstr. 6-8, D-94469 Deggendorf, Germany

Van Zyl, Ian

Siemens Milltronics Process Instruments Inc.

1954 Technology Drive, Peterborough, Ontario K9J 7B1, Canada

\section{Introduction}

The measurement of level in a tank, bunker or reservoir is one of the most essential measurements in the material and process industry. There are many applications using level measurements:

- Chemical and petrochemical

- Food and beverage

- Water and waste-water

- Pharmaceutical

- Mining and offshore

- Cement

- Transportation

\section{Methods}

The physical methods are various and can be divided as in the VDI-VDE recommendation "Level measurement of liquids and solids" [1] as follows:

- Visual

- Float

Sight glasses also with parallel vessel, diptape, dipstick.

Determining the position of a body floating on the liquid's surface.

- Displacer

Measuring the buoyancy force of a body partly dipped in the liquid.

- Ground pressure

Measuring the hydrostatic pressure at the bottom of the vessel.

- Weighing

Measuring the weight of the vessel and considering the product's density and the horizontal crosssection of the vessel.

- Measuring by retarding and restricting movements

Level switches by damping mechanical vibration or retarding rotary motions, also plumbing method.

- Measuring by electrical resistance

Measuring the resistance of the conductive liquid between two electrodes in the tank, also potentiometric method or using a resistor chain activated by the filled-in product.

- Capacitive and admittance measuring Measuring the capacitance of a dielectric product between two electrodes or the admittance of an isolated electrode system surrounded by the filling liquid if it is electrically conductive. For details see section 4 .

- Measuring by heat dissipation Method based on the different thermal conductivities of the gas phase above the liquid and of the liquid itself, detected by a heated sensor.

- Radiometric

Method detecting the absorption of gamma rays transmitted through the tank or its backscattering, influenced by the filling liquid that gives a higher absorption than the gas phase. The measuring equipment is usually mounted completely outside the tank, therefore it is a real non-contact measurement.

- Sonic and ultrasonic measuring

Measuring the empty space above the product's level by the propagation time of acoustic waves reflected on the surface. Or by transmission of waves from the tank bottom travelling through the liquid. For details see section 3.1. 
- Measuring by microwaves

Here electromagnetic waves are propagated against the product's surface, reflected there and the time-of-flight measured. For details see section 3.2.

- Measuring by time domain reflectometry

Similar as microwave measurement, but the waves are guided by a transmission line dipped into the product to be measured. It has advantages over the "free-space" microwave method on low reflective products or for interface measurements. For details see section 3.3.

- Optical measuring

Here also a propagation time measurement with optical waves is possible in clean environments or a triangulation method, but mostly optical methods are used as level switches by absorption, reflection or refraction of light at the sensor in contact with a liquid.

Some of these physical principles have been very well established for a long period, like the mechanical methods such as visual, float, displacer, ground pressure, weighing, radiometric and measuring by retarding and restricting movements. They still have their place where special or difficult process situations exist, like easy handling, missing electrical energy, high pressure etc.

In the following the "more modern" methods will be handled that are in continuous technical progress, mainly by advances in electronic components.

\section{Time-of-flight measurements}

An indirect measurement of level is evaluating the time-of-flight of a wave propagating through the atmosphere above the liquid or solid. The level is measured indirectly by the difference between the fixed tank height and the distance to the surface of the product in the tank.

The increasing demand of industry for non-intrusive continuous level gauging systems has been instrumental in accelerating the development of technologies using time-of-flight measurements. Though different types of physical waves (acoustic or electromagnetic) are applied, the principle of all these methods is the same: A signal is emitted as a wave towards the product, reflected at its surface and received by a sensor, which in many cases is the same, e.g. the ultrasonic piezoelectric transducer or the radar antenna. Figure 1 demonstrates the principle of operation on the example of a radar system.

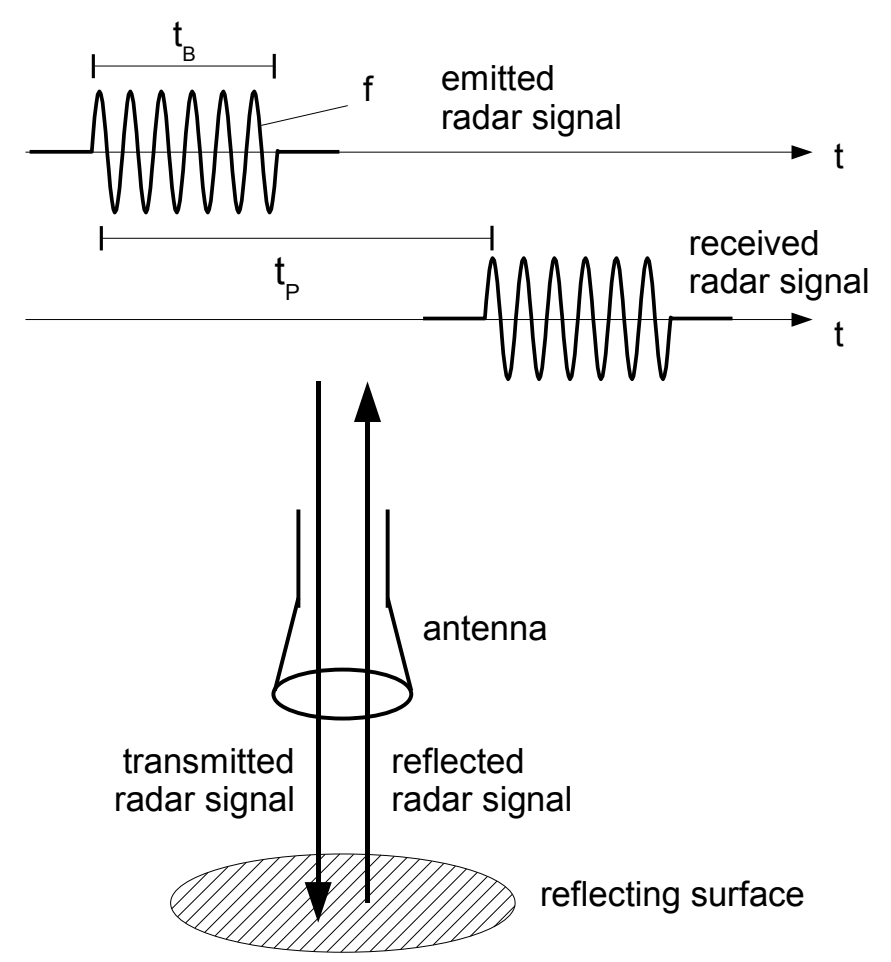

Figure 1: Principle of a pulsed time-of-flight level measurement [1] 
The measuring system evaluates the time-of-flight $t_{\mathrm{P}}$ of the signal:

$$
t_{P}=\frac{2 d}{v}
$$

where $d$ is the distance to the reflecting surface and $v$ the propagation velocity of the waves.

In principle all types of waves propagating through the gas phase above the liquid's or solid's level (or propagating through the product) and being reflected at the product's surface are suitable for time-of-flight measurements:

\begin{tabular}{|l|l|l|l|}
\hline Physical wave type & Used frequencies $f$ & Propagation velocity $v$ in air & See section \\
\hline Ultrasonic/acoustic & $13-6000 \mathrm{kHz}$ & $340 \mathrm{~m} / \mathrm{s}$ & 3.1 \\
\hline Microwaves/electromagnetic & $4-85 \mathrm{GHz}$ & $3 \times 10^{8} \mathrm{~m} / \mathrm{s}$ & 3.2 \\
\hline TDR/guided electromagnetic & $0-5 \mathrm{GHz}$ & $3 \times 10^{8} \mathrm{~m} / \mathrm{s}$ & 3.3 \\
\hline Laser/optical/infra-red & $200-800 \mathrm{THz}$ & $3 \times 10^{8} \mathrm{~m} / \mathrm{s}$ & --- \\
\hline
\end{tabular}

Table 1: Wave types for time-of-flight measurements

\subsection{Ultrasonic}

Ultrasonic waves are longitudinal acoustic waves with frequencies above $20 \mathrm{kHz}$. Ultrasonic needs a propagation medium, which for level measurements is the atmosphere above the product being measured or the liquid itself (when measuring from the tank bottom). The sound velocity of about $340 \mathrm{~m} / \mathrm{s}$ in air is highly dependent on temperature and composition of the gas. In vacuum, ultrasonic waves cannot propagate. In practice, the reflection ratio is nearly $100 \%$ at the product's surface, e.g. at transitions gas/liquid or gas/solid. Piezoelectric transducers are utilized as emitter and detector for ultrasonic waves, a membrane coupling it to the atmosphere. Level gauging is also possible with the sound coupled into the liquid, even through the tank wall, and propagating towards the surface where it is reflected.

\subsection{Radar}

The radar method uses high-frequency electromagnetic waves emitted and received by an antenna. The preferred frequencies according to ETSI EN 302372 [8] and ETSI TR 102601 are (Table 2):

\begin{tabular}{|l|l|l|}
\hline Frequency band & $\begin{array}{l}\text { Frequencies for closed tanks } \\
\text { acc. ETSI EN } 302372\end{array}$ & $\begin{array}{l}\text { Frequencies for open applications } \\
\text { acc. ETSI TR 102 601 (in preparation) }\end{array}$ \\
\hline C band & $4.5 \mathrm{GHz}$ to $7.0 \mathrm{GHz}$ & $6.0 \mathrm{GHz}$ to $8.5 \mathrm{GHz}$ \\
\hline X band & $8.5 \mathrm{GHz}$ to $10.6 \mathrm{GHz}$ & ---- \\
\hline K band & $24.05 \mathrm{GHz}$ to $27.0 \mathrm{GHz}$ & $24.05 \mathrm{GHz}$ to $26.5 \mathrm{GHz}$ \\
\hline V band & $57 \mathrm{GHz}$ to $64 \mathrm{GHz}$ & $57 \mathrm{GHz}$ to $64 \mathrm{GHz}$ \\
\hline W band & $75 \mathrm{GHz}$ to $85 \mathrm{GHz}$ & $75 \mathrm{GHz}$ to $85 \mathrm{GHz}$ \\
\hline
\end{tabular}

Table 2: Frequencies for level probing radar applications

Current systems are available in the C-, X- and K-band. The higher frequencies would give smaller antennas and better applicability in smaller vessels or on solids in future.

There are two dominant radar methods used for level gauging:

- Pulse radar

- Frequency modulated continuous wave (FMCW) radar

The first emits short microwave pulses (see figure 1), propagating with the velocity of light $c$, and measures the time-of-flight $t_{\mathrm{P}}$ when receiving the signal again:

$$
d=\frac{c t_{P}}{2}
$$

The FMCW radar uses a signal with constant amplitude swept linearly in frequency with $\Delta f$ within a time period $T$ (Figure 2). Mixing the received signal with the emitted signal results in a low frequency $f$ that is proportional to the distance $d$ :

$$
f=t_{P} \frac{\Delta f}{T}=\frac{2 d}{c} \frac{\Delta f}{T}
$$




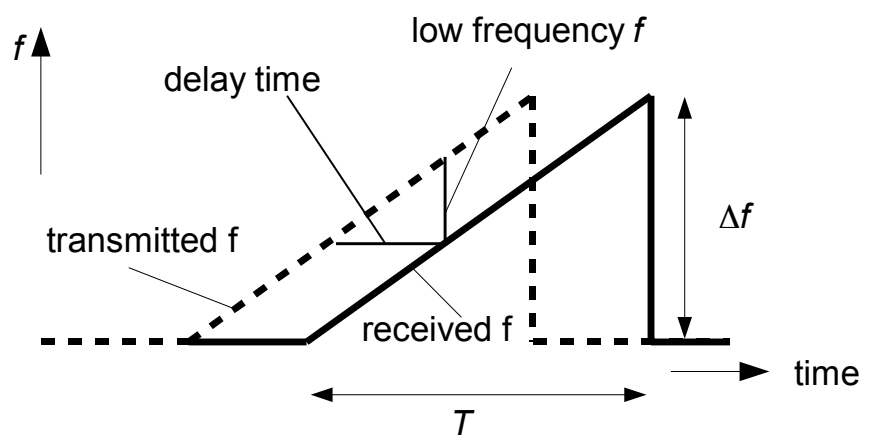

Figure 2: Frequency vs. time for an FMCW radar

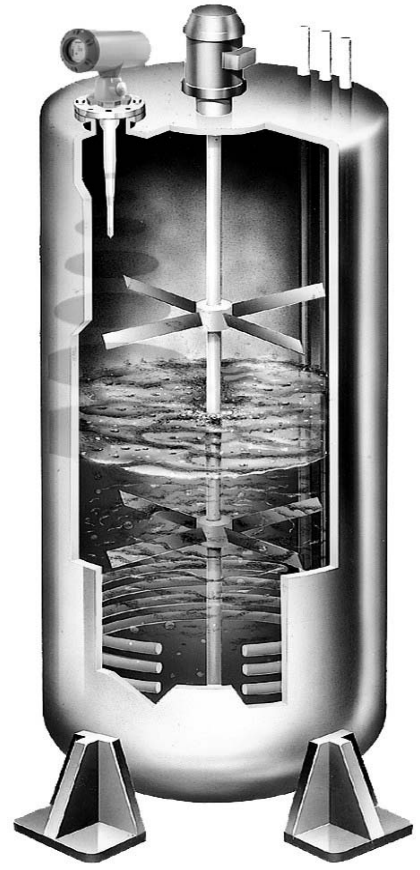

Figure 3: Process tank [9]

The radar method is also able to detect multiple reflections. From the signal that contains the distance information the most likely reflection from the surface has to be extracted. But this may be disturbed by false echoes from the antenna, pipes, agitator and other installations in the tank (Figure 3), or by multiple and tank wall reflections. This distinction of several targets is the most challenging task for digital signal evaluation.

On the other side quite small reflections from far targets have to be separated, requiring a good S/N ratio and an appropriate evaluation algorithm. For a pulse radar a time expansion technique and for an FMCW radar the FFT (Fast Fourier Transform) is mostly used to fulfil these requirements.

The reflected microwave power at the target - the liquid's or solid's surface - depends on the relative permittivity $\varepsilon_{r}$ of the medium in the tank. The power reflection factor $R$ may be quite poor as shown by the following equation and table 3 :

$$
R=\left(\frac{\sqrt{\epsilon_{r}}-1}{\sqrt{\epsilon_{r}}+1}\right)^{2}
$$

\begin{tabular}{|l|c|ll|}
\hline Medium & $\varepsilon_{r}$ value & \multicolumn{2}{|c|}{ Reflectivity $R$} \\
\hline water @ $5 \mathrm{GHz}$ & 75 & $63 \%$ & $-2 \mathrm{~dB}$ \\
\hline water @ $80 \mathrm{GHz}$ & 12 & $30 \%$ & $-5 \mathrm{~dB}$ \\
\hline alcohol & 9 & $25 \%$ & $-6 \mathrm{~dB}$ \\
\hline liquid sulphur & 3.5 & $9 \%$ & $-10 \mathrm{~dB}$ \\
\hline petrol & 2 & $3 \%$ & $-15 \mathrm{~dB}$ \\
\hline liquid carbon dioxide & 1.6 & $1.4 \%$ & $-19 \mathrm{~dB}$ \\
\hline plastic pellets & 1.3 & $0.4 \%$ & $-24 \mathrm{~dB}$ \\
\hline
\end{tabular}

Table 3: Reflection characteristics of some media

Additionally wave scattering may appear from a solid's rough surface or foam or disturbed liquid surface, giving i.e. $20 \mathrm{~dB}$ more attenuation. Thus, up to more than $40 \mathrm{~dB}$ reflection losses have to be accounted for when calculating the received signal and the system's signal-to-noise ratio. 


\subsection{TDR}

An alternative method called "Time Domain Reflectometry" (TDR) uses electromagnetic pulses to propagate them in a kind of cable formed of one or two parallel conductors. Figure 4 illustrates the operation with a line dipped into the liquid or bulk material. Where the dielectric permittivity of the surrounding medium changes, part of the wave is reflected. This method can be applied to interface measurements too. The right side of figure 4 and part (b) show the signals in an application with a twophase product.

(a)

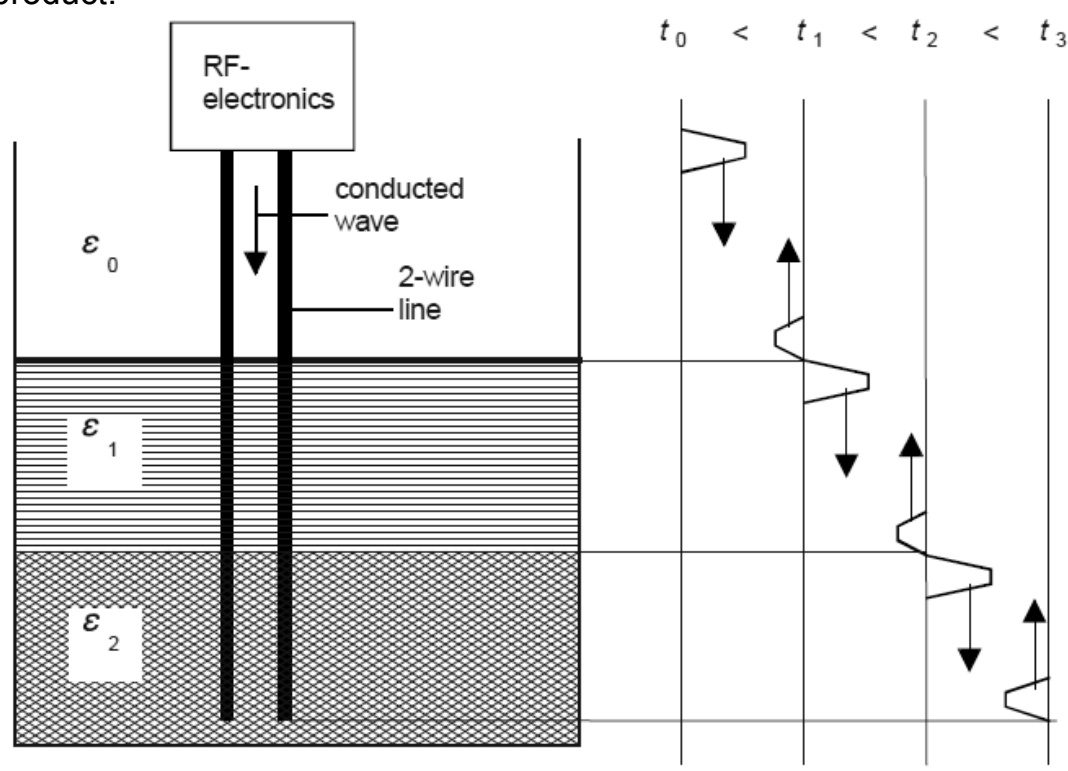

(b)

Signal at the sensor:

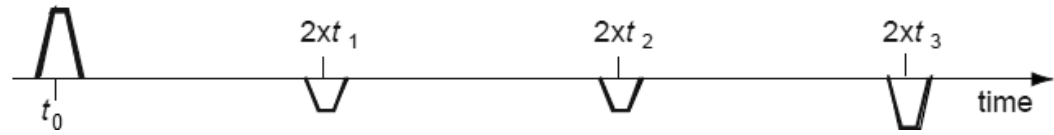

Figure 4: Function of a TDR system for interface measurements [6]

\section{Capacitive and admittance measurements}

In most applications, a rod electrode is arranged vertically in the tank. The electrode can be non-insulated if the liquid is non-conductive, or may be insulated. The metallic vessel acts as a reference electrode. The measured capacitance $C$ depends on the level $L$ and the permittivity $\varepsilon_{2}$ of the product. Figure 5 shows such an electrode concentrically mounted on a cylindrical tank.

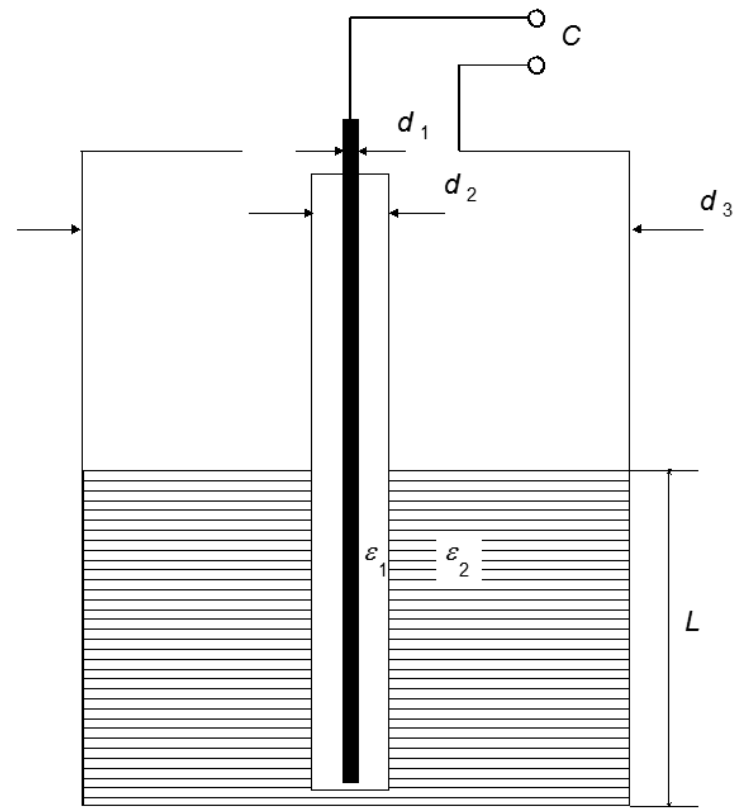

Figure 5: Configuration of a capacitive level measurement [2]

$$
C=\frac{2 \pi \epsilon_{0} L}{\frac{1}{\epsilon_{1}} \ln \frac{d_{2}}{d_{1}}+\frac{1}{\epsilon_{2}} \ln \frac{d_{3}}{d_{2}}}
$$

Calculation of the electrode capacitance 
For such a rotationally symmetrical configuration, the capacitance $C$ of an insulated electrode changes with level $L$ according to above equation.

For the measurement of conductive liquids, the determination of only the capacitive resistance would result in errors, in this case a method measuring the complex impedance or admittance is more precise and reliable.

Some newer systems adapt the measuring frequency inversely proportional to the capacitance (inverse frequency shift) in order to achieve a better resolution on low capacitances.

\section{Conclusion}

A huge variety of different technologies exist to measure level: From well established techniques, even simple mechanical ones, up to modern physical solutions with high-tech electronics supported by advanced computer technology.

The user has to decide in favour of the best suitable measuring method and choose a specific instrument for his application. In order to assist him in his choice, some manufacturer-independent overviews and decision tables are available. One is the VDI/VDE recommendation 3519 [1] where each technology is described in detail including its limitations. Figure 6 shows an extract of the decision table.

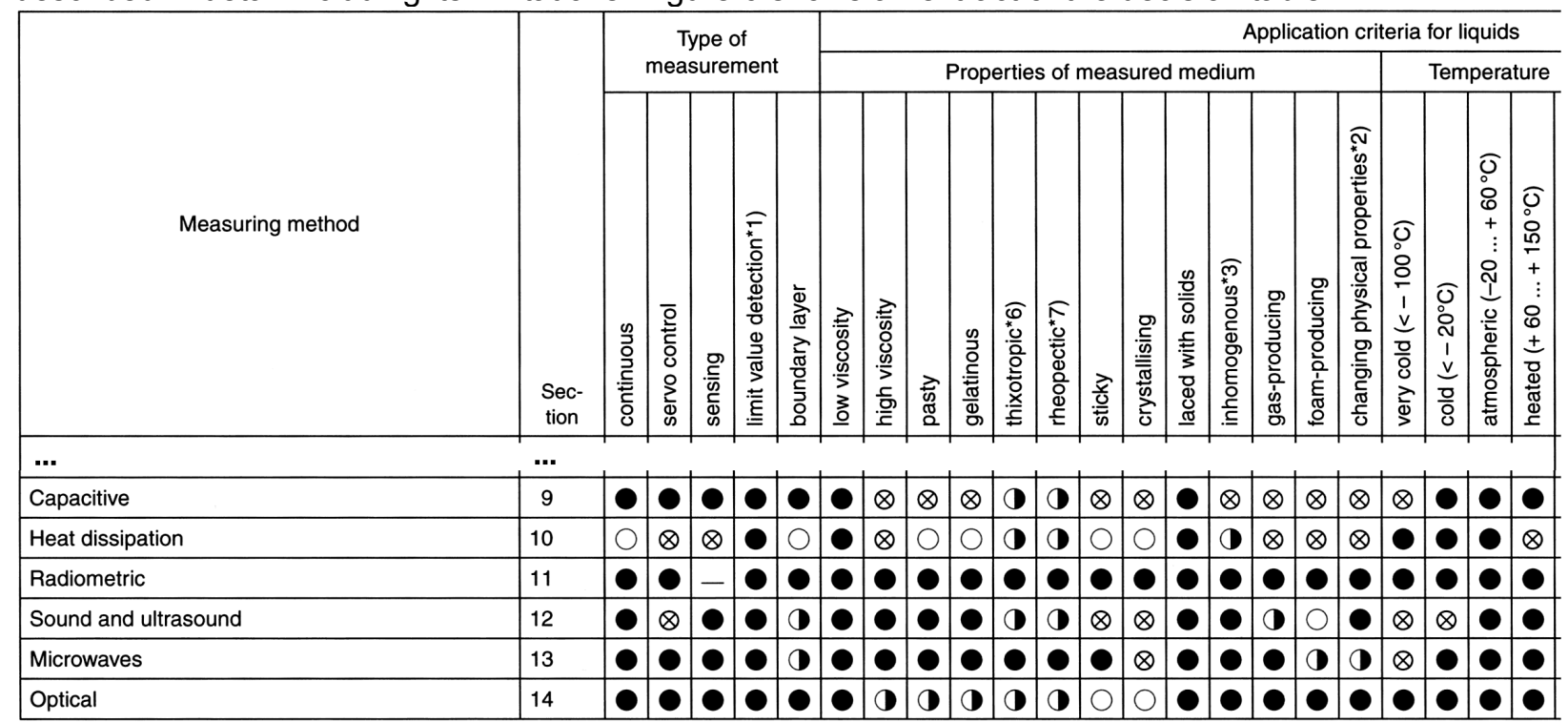

Figure 6: Measuring methods, overview extract [1] - suitable partially suitable $\otimes$ under conditions - NA

\section{References:}

[1] VDI/VDE 3519:2002. Füllstandmesstechnik, Berlin: Beuth Verlag (under revision; new edition in 2010)

[2] J. G. Webster: The Measurement, Instrumentation and Sensors Handbook, CRC Press LLC, 1999

[3] D.R. Gillum: Industrial Pressure, Level \& Density Measurement. Instrument Society of America, 1995

[4] K.W. Bonfig, Technische Füllstandsmessung und Grenzstandskontrolle, expert-Verlag, 1990

[5] D. Brumbi, "Industrial Level Sensing with Radar", Frequenz - Journal of RF-Engineering and Telecommunications, Volume 60, January/February 2006

[6] D. Brumbi: Grundlagen der Radartechnik zur Füllstandmessung, 4. Auflage. www.brumbi.de/Radartechnik.pdf, 2003

[7] D. Brumbi, "Low Power FMCW Radar System for Level Gaging", 2000 IEEE MTT-S Symposium Digest, Volume 3, p. 1559-1562

[8] ETSI: Electromagnetic Compatibility and Radio Spectrum Matters (ERM); Short Range Devices (SRD); Equipment for Detection and Movement; Tank Level Probing Radar (TLPR) Operating in the frequency bands $5,8 \mathrm{GHz}, 10 \mathrm{GHz}, 25 \mathrm{GHz}, 61 \mathrm{GHz}$ and $77 \mathrm{GHz}$ (ETSI EN 302 372). Sophia Antipolis, ETSI, 2006-04

[9] Siemens Milltronics Process Instruments Inc.: Level Measurement, www.siemens-milltronics.com 